\title{
Action Seniors! - secondary falls prevention in community-dwelling senior fallers: study protocol for a randomized controlled trial
}

Teresa Liu-Ambrose ${ }^{1 *}$, Jennifer C Davis ${ }^{1,2}$, Chun Liang Hsu' ${ }^{1}$ Caitlin Gomez ${ }^{1}$, Kelly Vertes ${ }^{1}$, Carlo Marra ${ }^{3}$, Penelope M Brasher ${ }^{2}$, Elizabeth Dao ${ }^{1}$, Karim M Khan ${ }^{4}$, Wendy Cook ${ }^{5}$, Meghan G Donaldson², Ryan Rhodes ${ }^{7}$ and Larry Dian ${ }^{6}$

\begin{abstract}
Background: Falls are a 'geriatric giant' and are the third leading cause of chronic disability worldwide. About 30\% of community-dwellers over the age of 65 experience one or more falls every year leading to significant risk for hospitalization, institutionalization, and even death. As the proportion of older adults increases, falls will place an increasing demand and cost on the health care system. Exercise can effectively and efficiently reduce falls. Specifically, the Otago Exercise Program has demonstrated benefit and cost-effectiveness for the primary prevention of falls in four randomized trials of community-dwelling seniors. Although evidence is mounting, few studies have evaluated exercise for secondary falls prevention (that is, preventing falls among those with a significant history of falls). Hence, we propose a randomized controlled trial powered for falls that will, for the first time, assess the efficacy and efficiency of the Otago Exercise Program for secondary falls prevention.

Methods/Design: A randomized controlled trial among 344 community-dwelling seniors aged 70 years and older who attend a falls prevention clinic to assess the efficacy and the cost-effectiveness of a 12-month Otago Exercise Program intervention as a secondary falls prevention strategy. Participants randomized to the control group will continue to behave as they did prior to study enrolment. The economic evaluation will examine the incremental costs and benefits generated by using the Otago Exercise Program intervention versus the control.

Discussion: The burden of falls is significant. The challenge is to make a difference - to discover effective, ideally cost-effective, interventions that prevent injurious falls that can be readily translated to the population. Our proposal is very practical - the exercise program requires minimal equipment, the physical therapist expertise is widely available, and seniors in Canada and elsewhere have adopted the program and complied with it. Our innovation includes applying the intervention to a targeted high-risk population, aiming to provide the best value for money. Given society's limited financial resources and the known and increasing burden of falls, there is an urgent need to test this feasible intervention which would be eminently ready for roll out.
\end{abstract}

Trial registration: ClinicalTrials.gov Protocol Registration System: NCT01029171; registered 7 December 2009.

Keywords: Otago Exercise Program, Falls, Resistance Training, Executive Functions, Older Adults

\footnotetext{
* Correspondence: Teresa.ambrose@ubc.ca

'Aging, Mobility, and Cognitive Neuroscience Laboratory, Djavad

Mowafaghian Centre for Brain Health, University of British Columbia,

212-2177 Wesbrook Mall, Vancouver, BC V6T 1Z3, Canada

Full list of author information is available at the end of the article
} 


\section{Background}

Falls are a common geriatric syndrome [1] and are the third leading cause of chronic disability worldwide [2]. Falls impose significant risk for hospitalization, institutionalization, and even death [3-5]. About 30\% of community-dwellers over the age of 65 experience one or more falls every year [6], with half of these seniors experiencing recurrent falls. With the proportion of older adults increasing, falls will continue to place an increasing health and economic burden on the public health system.

Exercise can effectively reduce falls. Specifically, New Zealand researchers designed a physical therapist-delivered, progressive home-based strength and balance training program tailored for seniors [7-11]. This intervention - the Otago Exercise Program (OEP) - has demonstrated benefit in four randomized trials of community-dwelling seniors selected based on age alone (that is, $\geq 80$ years old) [7-11]. Only one of these four trials designated falls as the primary outcome [12] while the others focused on measures of falls risk. Hence, the OEP qualifies as primary falls prevention (that is, preventing falls among those without a history of falls). The Cochrane Collaboration [13] explicitly identifies the OEP as the exercise training program with the strongest evidence for falls prevention. Although the OEP is the exercise training program with the strongest evidence for primary falls prevention [13], no randomized controlled trial (RCT) powered for falls has evaluated the efficacy of the $\mathrm{OEP}$ as a secondary falls prevention (that is, preventing falls among those with a history of falls) strategy. Hence, a rigorously designed RCT with falls as the primary outcome is an essential next step to determine the role of OEP in preventing falls among senior men and women with a significant history of falls. Previous research has demonstrated that the best value for money of various falls prevention strategies comes from targeting high-risk groups [14].

Improved physiological function is the generally accepted mechanism underlying the effectiveness of the OEP in reducing falls [8]. However, in a meta-analysis of four OEP randomized trials, falls were significantly reduced by $35 \%$ while postural sway significantly improved by only $9 \%$ and there was no significant improvement in knee extension strength [11]. Hence, the OEP may reduce falls via mechanisms other than improved physiological function. Specifically, we have demonstrated proof-of-concept data suggesting that improved cognitive function may be a very important mechanism by which the OEP reduces falls [15].

Within the multiple domains of cognitive function, reduced executive functions are associated with falls [16-20]. Executive functions are higher order cognitive processes that control, integrate, organize, and maintain other cognitive abilities [21]. Executive functions decline substantially with aging [22]. Importantly, reduced executive functions are prevalent among healthy, community-dwelling seniors with intact global cognitive function (that is, Mini-Mental State Examination (MMSE) score $\geq 24 / 30$ ) $[23,24]$. This is not surprising given that many of the pathological changes (for example, white matter lesions) associated with reduced executive functions are prevalent but clinically silent [25]. Our proof-of-concept study provided preliminary evidence that the OEP may improve executive functions in senior fallers [15]. Given the association between executive functions, exercise, and falls, we hypothesize that improved executive functions may be an important mechanism by which exercise reduces falls. However, this hypothesis is yet to be tested. Furthermore, our proof-of-concept study did not have the sample size to explore whether the observed change in cognitive function was a mediator of the benefit of the OEP.

Thus, we propose a 12-month RCT among communitydwelling seniors aged 70 years and older who attend a secondary falls prevention clinic to assess the efficacy and the cost-effectiveness of the OEP as a secondary falls prevention strategy. Further, we aim to explore the relative importance of both physiological and cognitive factors to falls reduction. Given the immense health and financial burden imposed by falls, our proposed RCT could have significant impact on the health of Canadian seniors and the Canadian health care system.

\section{Methods \\ Design}

We propose a RCT of 344 community-dwelling senior with a history of falls (that is, one or more falls in the past 12 months), aged 70 and older. Participant randomized to the OEP intervention group will receive the intervention for 12-months. There will be three measurement sessions with monthly monitoring (Figure 1).

\section{Setting}

All participants will be recruited from the Falls Prevention Clinic at Vancouver General Hospital (www.fallclinic. com).

\section{Participants}

All participants attending the Falls Prevention Clinic have sustained one or more falls in the past 12 months. Referrals to the Falls Prevention Clinic are from health care professionals (for example, physicians) for those who sought medical attention for their fall. Patients who attend the Falls Prevention Clinic receive falls risk factor assessment followed by a comprehensive geriatric assessment. The Falls Prevention Clinic care pathway is based on the American Geriatrics Society/British Geriatrics Society/ American Academy of Orthopedic Surgeons Falls Prevention Guidelines [26] (which is hereafter referred to as "standard of care"). 


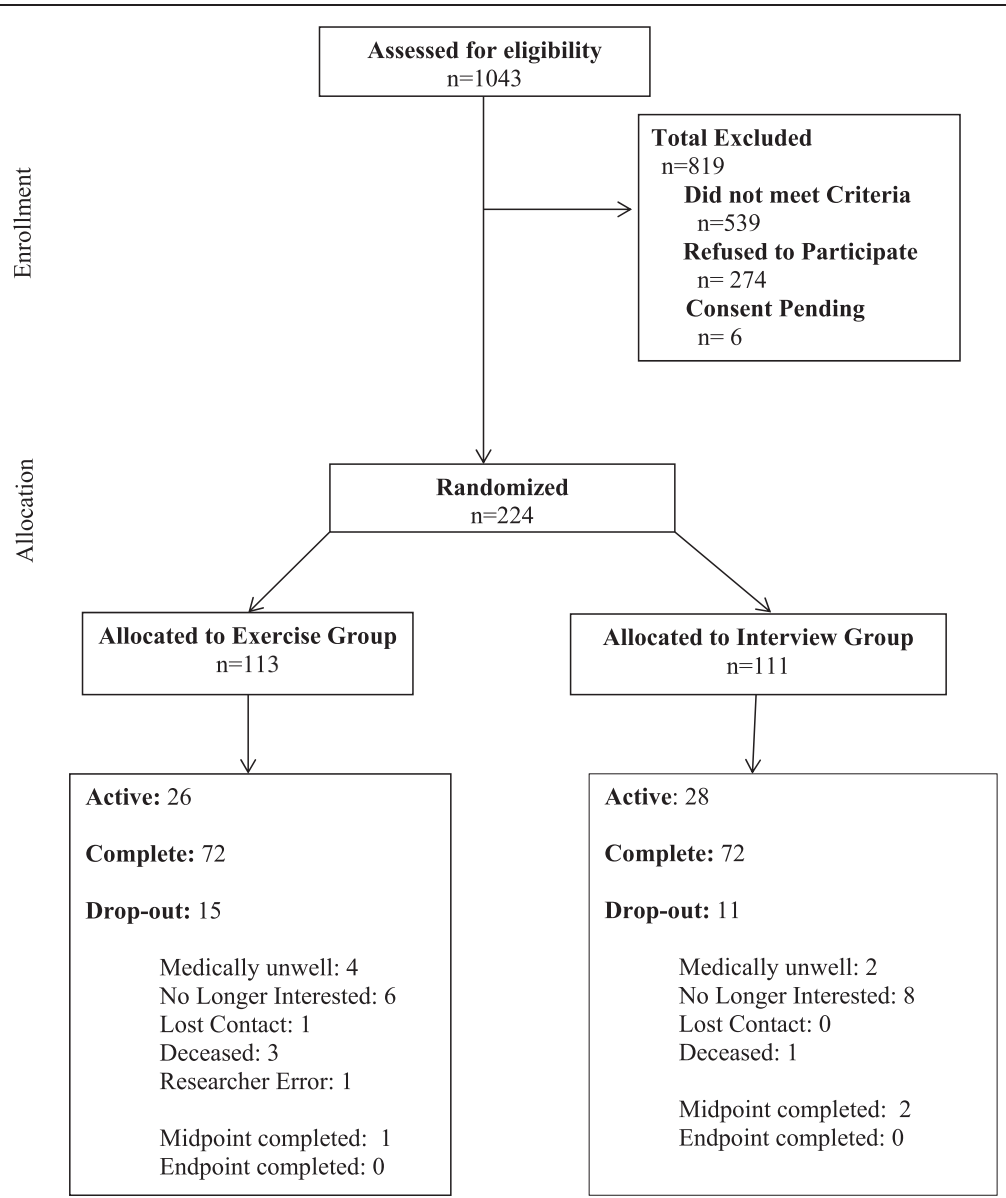

Figure 1 Overview of the flow of participants through the Action Seniors! trial.

Charts from the clinic will be reviewed on a weekly basis to identify eligible participants. Those who appear eligible based on detailed chart review will be mailed an information package and asked to call a research assistant if they are interested in participating in the study. When phone contact generates a person's agreement to participate, a research assistant will follow-up with a home visit. During this home visit, the consent form will be reviewed. Once written informed consent is obtained, the research assistant will complete the baseline assessment. Upon completion of the assessment, the research assistant who will remain blinded to group allocation will contact the research coordinator who will access the central randomization service to reveal the treatment allocation.

\section{Eligibility}

\section{Inclusion criteria}

1. Adults $\geq 70$ years referred by a medical professional to the Falls Prevention Clinic as a result of seeking medical attention for a non-syncopal fall in the previous 12 months

2. Understands, speaks, and reads English proficiently

3. MMSE [27] score $\geq 24 / 30$

4. A Physiological Profile Assessment (PPA; Prince of Wales Medical Research Institute, Sydney, Australia) [28] score of at least 1.0 standard deviation above age-normative value

or

Timed Up and Go (TUG) test [29] performance of greater than 15 seconds

or

one additional non-syncopal fall in the previous 12 months

5. Expected to live greater than 12 months (based on the geriatricians' expert opinion);

6. Living in the Greater Vancouver area

7. Community-dwelling (that is, not residing in a nursing home, extended care unit, or assisted-care facility)

8. Able to walk 3 meters with or without an assistive device

9. Able to provide written informed consent 


\section{Exclusion criteria}

1. Previously diagnosed with or suspected (by the geriatrician) to have neurodegenerative disease (for example. Parkinson's disease)

2. Previously diagnosed with or suspected (by the geriatrician) to have dementia (of any type)

3. Had a stroke

4. Have a history indicative of carotid sinus sensitivity (that is, syncopal falls)

Ethical approval has been obtained from the Vancouver Coastal Health Research Institute (V10-70171, 11 May 2004) and the University of British Columbia's Clinical Research Ethics Board (H04-70171, 11 May 2004).

\section{Power calculation}

The primary outcome is self-reported number of falls over the 12-month follow-up period. The sample size calculation employs a negative binomial regression model [30] to account for the overdispersion typical of falls data. Assuming an average fall rate in the control group of 1.0 falls per year, an average follow-up of 0.9 years and an overdispersion parameter, $\phi$, of 1.6 , we require 163 seniors per group to have $80 \%$ power to detect a $35 \%$ relative reduction in fall rate (that is, 1.0 versus 0.65 falls per year). To accommodate a complete loss to follow-up rate of $5 \%$ (that is, no fall diaries returned) we will recruit a total of 344 seniors (that is, 172 per group). The estimate of the control fall rate comes from the pooled analysis of four trials in a similar population [11]. The estimate of the overdispersion parameter comes from analysis of the data in Table two of Shumway-Cook [31] which yields $\phi=1.6$. The estimate for the average length of follow-up is based on our previous proof-of-concept study conducted locally in the same patient population in Greater Vancouver [15,32]. Only one of 74 participants returned no fall diaries so our estimate of a $5 \%$ complete loss to follow-up rate is conservative [32].

\section{Measurements}

Baseline measurements will be obtained prior to randomization. There will be three measurement sessions: baseline, 6 months, and 12 months.

\section{Falls prevention clinic visit}

The measurements listed below are acquired as part of the Falls Prevention Clinic visit and will be collected as the participants' baseline values upon informed consent.

Anthropometry Standing height is measured as stretch stature to $0.1 \mathrm{~cm}$ per standard protocol. Weight will be measured to $0.1 \mathrm{~kg}$ on a calibrated digital scale.
Geriatrician examination All patients undergo a comprehensive geriatrician assessment based on the American Geriatrics Society/British Geriatrics Society/American Academy of Orthopedic Surgeons Falls Prevention Guidelines [26].

General health, falls history, and socioeconomic status General health, falls history in the last 6 months [33], and socioeconomic status are ascertained by questionnaires.

Global cognitive function Global cognitive function is assessed using both the MMSE [27] and the Montreal Cognitive Assessment (MoCA) [34]. The MoCA is a brief 30-point screening tool for mild cognitive impairment [34] with high sensitivity and specificity. Specifically, it is more sensitive than the MMSE in detecting mild cognitive impairment. Using a cut-off score of 26, the MMSE had a sensitivity of $18 \%$, whereas the MoCA detected $90 \%$ of individuals with mild cognitive impairment [34].

Balance and mobility General balance and mobility is assessed with the: 1) Short Physical Performance Battery [35]; and 2) the TUG test [29]. For the Short Physical Performance Battery, participants are assessed on performances of standing balance, walking, and sit-to-stand. Each component is rated out of four points, for a maximum of 12 points. Poor performance on this scale predicts subsequent disability [35]. For the TUG test, participants are instructed to rise from a standard chair, walk a distance of 3 meters, turn, walk back to the chair and sit down. A TUG performance time of $\geq 13.5$ seconds correctly classified persons as fallers in $90 \%$ of cases [36].

Physiological falls risk We use the PPA [28] to assess physiological falls risk. The PPA is a valid and reliable tool for falls risk assessment. Based on the performance of five physiological domains (postural sway, hand reaction time, quadriceps strength, proprioception, and edge contrast sensitivity), the PPA computes a falls risk score for each individual and this measure has a $75 \%$ predictive accuracy for falls in older people [28]. A PPA z-score of 0 to 1 indicates mild risk, 1 to 2 indicates moderate risk, 2 to 3 indicates high risk, and 3 and above indicates marked risk [37].

Mood We use the 15-item Geriatric Depression Scale $[38,39]$ to screen for depression. The Geriatric Depression Scale specifically assesses for depressed mood in older people and a score of 5 and greater indicates depression [39].

Co-morbidity The Functional Co-morbidity Index was calculated to estimate the degree of co-morbidity associated with physical functioning [40]. 
Instrumental Activities of Daily Living scale The Lawton and Brody [41] Instrumental Activities of Daily Living Scale screens for impaired instrumental activities of daily living. This scale subjectively assesses ability to telephone, shop, prepare food, housekeep, do laundry, handle finances, be responsible for taking medication, and determining mode of transportation.

\section{Baseline home visit}

The following additional measures will be acquired during the home visit when written consent is obtained. The maximum time lag between the baseline Falls Prevention Clinic visit and the home visit is 1 month.

Falls-related self-efficacy Falls-related self-efficacy will be assessed by the Activities-Specific Balance Confidence (ABC) Scale. The 16-item ABC Scale [42] assesses fallsrelated self-efficacy with each item rated from $0 \%$ (no confidence) to $100 \%$ (complete confidence). The ABC Scale score is correlated with other measures of selfefficacy, distinguishes between individuals of low and high mobility, and corresponds with balance performance measures $[43,44]$.

Physical activity level Current physical activity level will be assessed by the valid and reliable Physical Activities Scale for the Elderly questionnaire $[45,46]$. This 12-item scale measures the average number of hours per day spent participating in leisure, household, and occupational physical activities over the previous 7-day period.

Executive functions There is no unitary executive function - rather, there are distinct processes. Thus, no single measure of executive function can adequately tap the construct in its entirety. Within the context of our proposal, we refer to work by Miyake and colleagues [47] who identified three key executive processes: 1) set shifting; 2) updating (or working memory); and 3) selective attention and conflict resolution (or response inhibition). Set shifting requires one to go back and forth between multiple tasks or mental sets [47]. Updating involves monitoring incoming information for relevance to the task at hand and then appropriately updating the informational content by replacing old, no longer relevant information with new incoming information. Conflict resolution involves deliberately inhibiting dominant, automatic, or prepotent responses. We will assess: 1) set shifting using the Trail Making Test (Part A and B) [48]; 2) updating (that is, working memory) using the verbal digits forward and backward test [49]; and 3) response inhibition using the Stroop Colour-Word Test [50]. These standardized neuropsychological tests are sensitive to age- $[48,51]$ and intervention-related changes [52-56]. Information processing speed will be indexed using the Digit Symbol
Substitution Test [57]. For this task, participants are first presented with a series of numbers (1 to 9 ) and their corresponding symbols. They are asked to draw the correct symbol for any digit placed randomly in pre-defined series in 60 seconds. A higher number of correct answers in this time period indicates better executive functions and processing speed.

Verbal fluency Defined as the rate at which an individual can generate words, verbal fluency will be assessed using both the FAS test (which assesses phonemic verbal fluency) and the animal naming test (which assesses semantic verbal fluency) [48]. For the FAS verbal fluency test, participants will be asked to verbally generate as many words (excluding proper names) as they can starting with the letters "F", "A" and "S", each in 60 seconds [48]. The total number of words generated for all three letters will be used as the measure of performance. For the animal naming test, participants will be asked to generate a list of animal names in 60 seconds [48].

Health-related quality of life We will evaluate healthrelated quality of life using Euro-Qol-5D three level (EQ-5D-3 L) [58]. The EQ-5D ascertains health status according to the following domains: mobility, self-care, usual activities, pain, and anxiety/depression. We will calculate quality-adjusted life years using the weightings from each instrument to compare differences in the incremental cost-effectiveness ratios.

\section{Monthly measurement}

The following measures will be collected monthly by telephone: 1) current physical activity level as assessed by the Physical Activities Scale for the Elderly questionnaire; and 2) health-related quality of life as assessed by the Short Form 6D [59], EuroQol EQ-5D-3 L [58], and Health Utilities Index Mark 3 [60]. Strategies to promote adherence to the OEP exercises during these monthly phone calls will also occur.

Through monthly calendars and diaries, participants will be asked to provide the following information: 1 ) falls and adherence to the OEP (ascertainment of falls and adherence to the OEP will be documented on monthly calendars); and 2) health care resource utilization and costs (participants will complete monthly health care resource use diaries over the 12-month study period).

\section{Randomization}

Participants will be randomly assigned (1:1) to either the OEP (plus standard of care) group or the standard of care (control) group. The randomization sequence will be stratified by: 1) sex, as falls rate is different between men and women; and 2) geriatrician (LD and WC), as standard of care delivery may differ between physicians. 
Permuted blocks of varying size (for example, 2,4,6) will be employed. To ensure concealment of the treatment allocation, the randomization sequences will be generated and held by a central Internet randomization service.

\section{Planned trial interventions Otago Exercise Program intervention}

The OEP is an individualized home-based balance and strength retraining program $[8,61]$. It consists of the following strengthening exercises: knee extensor (4 levels), knee flexor (4 levels), hip abductor (4 levels), ankle plantarflexors (2 levels), and ankle dorsiflexors (2 levels). The balance retraining exercises consist of the following: knee bends (4 levels), backwards walking (2 levels), walking and turning around (2 levels), sideways walking (2 levels), tandem stance (2 levels), tandem walk (2 levels), one leg stand (3 levels), heel walking (2 levels), toe walking (2 levels), heel toe walking backwards (1 level), and sit to stand (4 levels).

Licensed physical therapists will deliver the OEP after a standard training session with the research team. For each OEP participant, a physical therapist will visit the home and prescribe a set of suitable exercises from the OEP manual. The same physical therapist will return bi-weekly three additional times to make progressive adjustments to the exercise protocol according to the OEP manual. Each of these four visits in the first 2 months will take approximately 1 hour. The physical therapist's fifth visit will occur 6 months after the initial visit. During this last visit, the physical therapist will check that the OEP exercises are being done correctly and will also encourage the participant to continue with the exercise program. Overall, the participant is asked to perform the OEP balance and strength retraining exercises three times per week (approximately 30 minutes). In addition to the OEP manual, which contains a picture and description of each exercise, each participant will be provided with an adjustable cuff weight (in $0.9 \mathrm{~kg}$ increments; range $=0.9$ to $9 \mathrm{~kg}$ ) to be used with the OEP strength training exercises. Based on data from our proof-of-concept study [15], the OEP is safe for our target population; only 2 of the 36 OEP participants reported low back pain as adverse events.

\section{Standard of care}

Participants randomized to "standard of care" they receive standard of care - visits with a geriatrician.

\section{Adverse events monitoring}

A physician and a statistician external to the daily activities of this study will review and compile a report for all adverse events reported in the study on a monthly basis. They will stop the study if the adverse event data demonstrate any hazards of the intervention (for example, increased falls or fracture) based on the monthly report.

\section{Statistical analyses}

Our primary, secondary, and tertiary analyses will follow the intention-to-treat principle (that is, all individuals will be analyzed according to their group allocation regardless of compliance).

\section{Primary outcome}

The rate of falls (the primary outcome) will be compared between the two groups using a negative binomial regression model. The treatment assignment and stratification factors will be included in the model as covariates. Point and interval estimates for the rate ratio will be determined.

\section{Secondary outcomes}

We will conduct exploratory analyses on the secondary outcomes (PPA, TUG test and Short Performance Physical Battery). Given that a potential source of bias in this trial will result from patients being unblinded to their group allocation, group will be controlled for in all secondary analyses.

\section{Economic evaluation}

Our economic evaluation will examine the incremental costs and benefits generated by using the OEP intervention versus standard of care. The outcome of our cost effectiveness analysis is the incremental cost-effective ratio (ICER). By definition, an ICER is the difference between the mean costs of providing the competing interventions divided by the difference in effectiveness, where ICER $=\Delta$ cost $/ \Delta$ effect [62]. Both a cost-effectiveness analysis and a cost utility analysis will be performed. Based on the primary outcome of the RCT, we will determine the incremental cost of the OEP intervention per fall avoided, relative to standard treatment. We will also conduct a cost-utility analysis. In a cost-utility analysis, the primary outcome is the quality-adjusted life years. These are calculated based on the quality of life of a patient (measured using health utilities) in a given health state and the time spent in that health state. An important aspect of economic evaluations conducted alongside an RCT is how to deal with missing data due to attrition. We will follow recommendations by Oostenbrick and colleagues [63] and Briggs and colleagues [64], and the International Society for Pharmacoeconomics and Outcomes Research [65], in dealing with missing cost and effectiveness data. We will use a combination of imputation and bootstrapping to quantify uncertainty due to missing values. 


\section{Mediation analysis}

We will use path analysis - a special case of structural equation modeling where all variables are observed to investigate how physiological function and cognitive function mediate the effect of the intervention on the primary outcome (that is, falls). Using Mplus 5.1 (www.statmodel.com) we will fit a negative binomial regression model that includes one independent variable and mediator variables.

\section{Discussion}

Our interdisciplinary research team will use a multipronged approach to explore the utility of OEP among seniors at high risk of future falls. The proposed trial may have important public health, economic, and mechanistic implications.

\section{Public health}

The simple and proven exercise program (that is, the OEP) has already been implemented nationally in New Zealand. Therefore, if our study demonstrates the OEP is an efficacious and efficient (that is, cost-effective) secondary falls prevention program, our findings could be rolled out immediately by policy makers.

\section{Economic}

The parallel economic evaluation is particularly important because, if the intervention proved to be cost-effective compared with standard of care, it would provide a strong argument for the OEP in the target population even at a time of fiscal restraint. We highlight that this intervention, the OEP, already has manuals, websites, and educational material ready for a 'turn-key' operation.

\section{Mechanistic}

Better understanding of the primary mechanisms underlying the OEP (that is, our tertiary research objective) would increase our capacity to refine and develop novel interventions for secondary falls prevention for the aging population. If improved executive functions prove to play a significant role in falls reduction, it would be a major contribution to knowledge in this field.

\section{Trial status}

As of 1 December 2014 we have obtained ethical approval, have registered the trial and we have successfully recruited 227 participants. We will aim to complete recruitment by 2017 . One hundred and fifty four patients have completed 6-month follow-up, 133 have completed 12-month follow-up and 26 participants have dropped out. The median number of participation days for individuals who dropped out of the study was 103.5 .

\begin{abstract}
Abbreviations
ABC: Activities-Specific Balance Confidence; EQ-5D-3L: Euro-Qol 5D three level version; ICER: Incremental cost-effective ratio; MMSE: Mini-Mental State Examination; MoCA: Montreal Cognitive Assessment; OEP: Otago Exercise Program; PPA: Physiological Profile Assessment; RCT: Randomized controlled trial; TUG: Timed Up and Go.
\end{abstract}

\section{Competing interests}

The authors declare that they have no competing interests.

\section{Authors' contributions}

TLA wrote the grant application that was funded by the Canadian Institute for Health Research and jointly drafted the Action Seniors! Protocol. JCD contributed to writing the grant application that was funded by the Canadian Institute for Health Research and jointly drafted the Action Seniors! Protocol. CLH, CG, KV, and ED are part of the Action Seniors! Research team and critically reviewed the manuscript. CM and PMB contributed to writing the grant application that was funded by the Canadian Institute for Health Research and critically reviewed the Action Seniors! Protocol. WC critically reviewed the grant application that was funded by the Canadian Institute for Health Research and critically reviewed the Action Seniors! Protocol. KMK contributed to writing the grant application that was funded by the Canadian Institute for Health Research. MGD and RR critically reviewed the grant application that was funded by the Canadian Institute for Health Research. LD is the geriatrician at the Falls Prevention Clinic and part of the Action Seniors! Research team. LD critically reviewed the manuscript. The grant application formed the bases for the manuscript. All authors have read and approved the final manuscript.

\section{Acknowledgements}

This study is funded by the Canadian Institute for Health Research. TLA is a Canada Research Chair in Physical Activity, Mobility, and Cognitive Neuroscience, a Michael Smith Foundation for Health Research (MSFHR) Scholar, a Canadian Institutes of Health Research (CIHR) New Investigator, and a Heart and Stroke Foundation of Canada's Henry JM Barnett's

Scholarship recipient. JCD was funded by a CIHR and MSFHR Postdoctoral Fellowship. ED is funded by a CIHR Doctoral Award - Frederick Banting and Charles Best Canada Graduate Scholarship. CLH is funded by a PhD Alzheimer Society Research Program Award. These funding agencies did not play a role in study design. We obtained approval from UBC Clinical Ethics Review Board.

\section{Author details}

${ }^{1}$ Aging, Mobility, and Cognitive Neuroscience Laboratory, Djavad Mowafaghian Centre for Brain Health, University of British Columbia, 212-2177 Wesbrook Mall, Vancouver, BC V6T 1Z3, Canada. ${ }^{2}$ Centre for Clinical Epidemiology and Evaluation, Vancouver Coastal Health Research Institute \& University of British Columbia, 7th Floor, 828 West 10th Avenue, Research Pavilion, Vancouver, BC V5Z 1M9, Canada. ${ }^{3}$ School of Pharmacy, Memorial University of Newfoundland, Health Sciences Centre, St. John's, NL A1B 3V6, Canada. ${ }^{4}$ Centre for Hip Health and Mobility, Vancouver Coastal Health Research Institute \& University of British Columbia, 2635 Laurel St, Vancouver, BC V5Z 1M9, Canada. ${ }^{5}$ St Paul's Hospital, University of British Columbia, 1081 Burrard Street, Vancouver, BC V6Z 1Y6, Canada. ${ }^{6}$ Vancouver Coastal Health Research Institute, Room 3665, 910 West 10th Avenue, Vancouver, BC V5Z 1M9, Canada. ${ }^{7}$ Behavioural Medicine (BMED) Lab, School of Exercise Science, Physical and Health Education, University of Victoria, 3800 Finnerty Road, Victoria, BC V8P 5C2, Canada.

Received: 15 December 2014 Accepted: 12 March 2015 Published online: 10 April 2015

\section{References}

1. Inouye SK, Studenski S, Tinetti ME, Kuchel GA. Geriatric syndromes: clinical, research, and policy implications of a core geriatric concept. J Am Geriatr Soc. 2007:55:780-91. comments 794-6.

2. Murray C, Lopez A. Global and regional descriptive epidemiology of disability: incidence, prevalence, health expectancies, and years lived with disability. In: Murray C, Lopez A, editors. The global burden of disease. Boston: The Harvard School of Public Health; 1996. p. 201-46.

3. Pluijm S, Smit J, Tromp E, Stel V, Deeg D, Bouter L, et al. A risk profile for identifying community-dwelling elderly with a high risk of recurrent falling: results of a 3-year prospective study. Osteoporos Int. 2006;17:417-25. 
4. Formiga F, Navarro M, Duaso E, Chivite D, Ruiz D, Perez-Castejon JM, et al. Factors associated with hip fracture-related falls among patients with a history of recurrent falling. Bone. 2008;43:941-4.

5. Rockwood K, Stolee P, McDowell I. Factors associated with institutionalization of older people in Canada: testing a multifactorial definition of frailty. J Am Geriatr Soc. 1996;44:578-82.

6. Tinetti ME, Speechley M, Ginter SF. Risk factors for falls among elderly persons living in the community. N Engl J Med. 1988;319:1701-7.

7. Campbell A, Robertson M, Gardner M, Norton R, Buchner D. Falls prevention over 2 years: a randomized controlled trial in women 80 years and older. Age Ageing. 1999;28:513-8.

8. Campbell J, Robertson M, Gardner M, Norton R, Tilyard M, Buchner D. Randomised controlled trial of a general practice programme of home based exercise to prevent falls in elderly women. Br Med J. 1997;315:1065-9.

9. Robertson M, Devlin N, Gardner M, Campbell A. Effectiveness and economic evaluation of a nurse delivered home exercise programme to prevent falls. 1: Randomized controlled trial. Br Med J. 2001;322:697-701.

10. Robertson M, Gardner M, Devlin N, McGee R, Campbell J. Effectiveness and economic evaluation of a nurse delivered home exercise programme to prevent falls. 2: Controlled trial in multiple centres. Br Med J. 2001;322:701-4.

11. Robertson MC, Campbell AJ, Gardner MM, Devlin N. Preventing injuries in older people by preventing falls: a meta-analysis of individual-level data. J Am Geriatr Soc. 2002;50:905-11.

12. Skelton D, Dinan S, Campbell M, Rutherford O. Tailored group exercise (Falls Management Exercise - FaME) reduces falls in community-dwelling older frequent fallers (an RCT). Age Ageing. 2005;34:636-9.

13. Gillespie LD, Robertson MC, Gillespie WJ, Lamb SE, Gates S, Cumming RG, et al. Interventions for preventing falls in older people living in the community. Cochrane Database Syst Rev (Online). 2009;2:CD007146.

14. Davis JC, Robertson MC, Ashe MC, Liu-Ambrose T, Khan KM, Marra CA. Does a home-based strength and balance programme in people aged $>$ or $=80$ years provide the best value for money to prevent falls? A systematic review of economic evaluations of falls prevention interventions. Br J Sports Med. 2010;44:80-9.

15. Liu-Ambrose T, Donaldson MG, Ahamed Y, Graf P, Cook WL, Close J, et al. Otago home-based strength and balance retraining improves executive functioning in older fallers: a randomized controlled trial. J Am Geriatr Soc. 2008;56:1821-30.

16. Rapport LJ, Webster JS, Flemming KL, Lindberg JW, Godlewski MC, Brees JE, et al. Predictors of falls among right-hemisphere stroke patients in the rehabilitation setting. Arch Phys Med Rehabil. 1993;74:621-6.

17. Rapport LJ, Hanks RA, Millis SR, Deshpande SA. Executive functioning and predictors of falls in the rehabilitation setting. Arch Phys Med Rehabil. 1998;79:629-33.

18. Lord S, Fitzpatrick R. Choice stepping reaction time: a composite measure of fall risk in older people. J Gerontol. 2001;10:M627-32.

19. Lundin-Olsson L, Nyberg L, Gustafson Y. "Stops walking when talking" as a predictor of falls in elderly people. Lancet. 1997;349:617.

20. Anstey KJ, von Sanden C, Luszcz MA. An 8-year prospective study of the relationship between cognitive performance and falling in very old adults. J Am Geriatr Soc. 2006;54:1169-76.

21. Stuss DT, Alexander MP. Executive functions and the frontal lobes: a conceptual view. Psychol Res. 2000;63:289-98.

22. West RL. An application of prefrontal cortex function theory to cognitive aging. Psychol Bull. 1996;120:272-92.

23. Boone KB, Miller BL, Lesser IM, Hill E, D'Elia L. Performance on frontal lobe tests in healthy older individuals. Dev Neuropsychol. 1990;6:215-23.

24. Royall DR. Prevalence of executive control function (ECF) impairment among healthy non-institutionalized retirees: the Freedom House Study. Gerontologist. 1998;38S:314-5.

25. Thal DR, Del Tredici K, Braak H. Neurodegeneration in normal brain aging and disease. Sci Aging Knowledge Environ. 2004;2004:e26.

26. American Geriatrics Society. Guideline for the prevention of falls in older persons. J Am Geriatr Soc. 2001;49:664-72.

27. Folstein MF, Folstein SE, McHugh PR. "Mini-mental state": a practical method for grading the cognitive state of patients for the clinician. J Psychiat Res. 1975;12:189-98.

28. Lord S, Sherrington C, Menz H, Close J. A physiological profile approach for falls prevention. In: Lord SR, Sherrington C, Menz HB, Close JCT, editors. Falls in older people: risk factors and strategies for prevention. Cambridge: Cambridge University Press; 2001. p. 221-38.
29. Whitney JC, Lord SR, Close JC. Streamlining assessment and intervention in a falls clinic using the timed up and go test and physiological profile assessments. Age Ageing. 2005;34:567-71.

30. Signorini D. Sample size for Poisson regression. Biometrika. 1991;78:446-50.

31. Shumway-Cook A, Silver IF, LeMier M, York S, Cummings P, Koepsell TD. Effectiveness of a community-based multifactorial intervention on falls and fall risk factors in community-living older adults: a randomized, controlled trial. J Gerontol A Biol Sci Med Sci. 2007;62:1420-7.

32. Donaldson MG. Falls risk in frail seniors: clinical and methodological studies. Vancouver: University of British Columbia; 2007.

33. Cummings $S$, Nevitt M, Kidd S. Forgetting falls: the limited accuracy of recall of falls in the elderly. J Am Geriatr Soc. 1988;36:613-6.

34. Nasreddine ZS, Phillips NA, Bedirian V, Charbonneau S, Whitehead V, Collin I, et al. The Montreal Cognitive Assessment, MoCA: a brief screening tool for mild cognitive impairment. J Am Geriatr Soc. 2005;53:695-9.

35. Guralnik JM, Ferrucci L, Simonsick EM, Salive ME, Wallace RB. Lower-extremity function in persons over the age of 70 years as a predictor of subsequent disability. N Engl J Med. 1995;332:556-62.

36. Shumway-Cook A, Brauer S, Woollacott M. Predicting the probability for falls in community-dwelling older adults using the Timed Up \& Go Test. Phys Ther. 2000;80:896-903.

37. Lord SR, Menz HB, Tiedemann A. A physiological profile approach to falls risk assessment and prevention. Phys Ther. 2003;83:237-52.

38. Yesavage JA, Brink TL, Rose TL, Lum O, Huang V, Adey M, et al. Development and validation of a geriatric depression screening scale: a preliminary report. J Psychiatr Res. 1982;17:37-49.

39. van Marwijk HW, Wallace P, de Bock GH, Hermans J, Kaptein AA, Mulder JD. Evaluation of the feasibility, reliability and diagnostic value of shortened versions of the geriatric depression scale. Br J Gen Pract. 1995;45:195-9.

40. Groll DL, To T, Bombardier C, Wright JG. The development of a comorbidity index with physical function as the outcome. J Clin Epidemiol. 2005;58:595-602.

41. Lawton MP, Brody EM. Assessment of older people: self-maintaining and instrumental activities of daily living. Gerontologist. 1969;9:179-86.

42. Powell L, Myers A. The Activities-specific Confidence (ABC) scale. J Gerontol. 1995;50A:M28-34

43. Myers A, Powell L, Maki B, Holliday P, Brawley L, Sherk W. Psychological indicators of balance confidence: relationship to actual and perceived abilities. J Gerontol. 1996;51A:M37-43.

44. Myers A, Fletcher P, Myers A, Sherk W. Discriminative and evaluative properties of the Activities-specific Balance Confidence (ABC) scale. J Gerontol. 1998;53:M287-94.

45. Washburn RA, Smith KW, Jette AM, Janney CA. The Physical Activity Scale for the Elderly (PASE): development and evaluation. J Clin Epidemiol. 1993;46:153-62.

46. Washburn RA, McAuley E, Katula J, Mihalko SL, Boileau RA. The physical activity scale for the elderly (PASE): evidence for validity. J Clin Epidemiol. 1999;52:643-51.

47. Miyake A, Friedman NP, Emerson MJ, Witzki AH, Howerter A, Wager TD. The unity and diversity of executive functions and their contributions to complex "Frontal Lobe" tasks: a latent variable analysis. Cognit Psychol. 2000;41:49-100.

48. Spreen O, Strauss E. A compendium of neurological tests. 2nd ed. New York: Oxford University Press, Inc; 1998.

49. Wechsler D. Wechsler adult intelligence scale - revised. In: The Psychological Corporation. San Antonio, Texas: Harcourt Brace Jovanovich Inc; 1981.

50. Trenerry MR, Crosson B, DeBoe J, Leber WR. Stroop neuropsychological screening test manual. Lutz, FL: Psychological Assessment Resources; 1989.

51. Kramer AF, Hahn S, Gopher D. Task coordination and aging: explorations of executive control processes in the task switching paradigm. Acta Psychol (Amst). 1999;101:339-78.

52. Colcombe S, Kramer AF. Fitness effects on the cognitive function of older adults: a meta-analytic study. Psychol Sci. 2003;14:125-30.

53. Kramer AF, Hahn S, Cohen NJ, Banich MT, McAuley E, Harrison CR, et al. Ageing, fitness and neurocognitive function. Nature. 1999;400:418-9.

54. Colcombe SJ, Kramer AF, Erickson KI, Scalf P, McAuley E, Cohen NJ, et al. Cardiovascular fitness, cortical plasticity, and aging. Proc Natl Acad Sci U S A. 2004;101:3316-21.

55. Tanne D, Freimark D, Poreh A, Merzeliak O, Bruck B, Schwammenthal Y, et al. Cognitive functions in severe congestive heart failure before and after an exercise training program. Int J Cardiol. 2005;103:145-9.

56. Kubesch S, Bretschneider M, Freudenmann R, Weidenhammer M, Lehmann M, Spitzer $M$, et al. Aerobic endurance exercise improves executive functions in depressed patients. J Clin Psychiat. 2003;64:1005-12. 
57. Lezak MD. Neuropsychological assessment. 3rd ed. New York: Oxford University Press; 1995.

58. Dolan P. Modeling valuations for EuroQol health states. Med Care. 1997:35:1095-108.

59. Brazier J, Roberts J, Deverill M. The estimation of a preference-based measure of health from the SF-36. J Health Econ. 2002;21 (2):271-92.

60. Horsman J, Furlong W, Feeny D, Torrance G. The Health Utilities Index (HUI): concepts, measurement properties and applications. Health Qual Life Outcomes. 2003;1:54. doi:10.1186/1477-7525-1-54.

61. Gardner MM, Buchner DM, Robertson MC, Campbell AJ. Practical implementation of an exercise-based falls prevention programme. Age Ageing. 2001;30:77-83.

62. Drummond M, Manca A, Sculpher M. Increasing the generalizability of economic evaluations: recommendations for the design, analysis, and reporting of studies. Int J Technol Assess Health Care. 2005;21:165-71.

63. Oostenbrink JB, Al MJ, Rutten-van Molken MP. Methods to analyse cost data of patients who withdraw in a clinical trial setting. Pharmacoeconomics. 2003;21:1103-12.

64. Briggs AH, Lozano-Ortega G, Spencer S, Bale G, Spencer MD, Burge PS. Estimating the cost-effectiveness of fluticasone propionate for treating chronic obstructive pulmonary disease in the presence of missing data. Value Health. 2006;9:227-35.

65. Ramsey S, Willke R, Briggs A, Brown R, Buxton M, Chawla A, et al. Good research practices for cost-effectiveness analysis alongside clinical trials: the ISPOR RCT-CEA Task Force report. Value Health. 2005;8:521-33.

\section{Submit your next manuscript to BioMed Central and take full advantage of:}

- Convenient online submission

- Thorough peer review

- No space constraints or color figure charges

- Immediate publication on acceptance

- Inclusion in PubMed, CAS, Scopus and Google Scholar

- Research which is freely available for redistribution 